\title{
Difference independence of the Riemann zeta function
}

\author{
by \\ YiK-Man Chiang (Hong Kong) and \\ ShaO-Ji Feng (Beijing and Hong Kong)
}

1. Introduction. Let $\zeta(s)$ denote the Riemann zeta function, and $\Gamma(s)$ denote the Eular gamma function in the complex plane. These two important special functions are related by the functional equation (see $[7,16]$ )

$$
\zeta(1-s)=2^{1-s} \pi^{-s} \cos \left(\frac{1}{2} \pi s\right) \Gamma(s) \zeta(s) .
$$

A classical theorem of Hölder [6] states that $\Gamma(s)$ does not satisfy any algebraic differential equation whose coefficients are rational functions (i.e. any equation of the form $f\left(s, y, y^{\prime}, \ldots, y^{(n)}\right)=0$, where $n$ is a nonnegative integer and $f$ is a polynomial in $y, y^{\prime}, \ldots, y^{(n)}$ whose coefficients are rational functions of $s$ ). Other proofs of this theorem were given in $[3,9,11,12]$. Bank and Kaufman [1] generalized the theorem to coefficients being meromorphic functions $\phi$ with Nevanlinna characteristic (see (1.5) below for definition) satisfying $T(r, \phi)=o(r)$ as $r \rightarrow \infty$.

The question of the differential independence of $\zeta(s)$ was touched upon by Hilbert in 1900 (see [5]). He conjectured that $\zeta(s)$ and other functions of the same type do not satisfy algebraic differential equations with rational coefficients. It follows from Hilbert's report that, based on Hölder's theorem on the algebraic differential independence of $\Gamma(s)$, he could prove the algebraic differential independence of $\zeta(s)$. Hilbert's conjectures were proved in $[10,13]$ (see also $[14,15])$.

2000 Mathematics Subject Classification: 11M06, 39A05, 30D35.

Key words and phrases: Riemann zeta function, difference equation, Nevanlinna characteristic.

Research of Y. M. Chiang was supported in part by the Research Grant Council of the Hong Kong Administrative Region, China (HKUST6135/01P).

Work of S. J. Feng is supported by the National Natural Science Foundation of China (Grant No. 10501044), in part by the Research Grant Council of the Hong Kong Administrative Region, China (HKUST6135/01P) and HKUST PDF Matching Fund. 
It is well known that the gamma function satisfies the difference equation

$$
\Gamma(s+1)=s \Gamma(s) .
$$

A natural question to ask is whether $\zeta(s)$ satisfies any algebraic difference equation, or briefly: is $\zeta(s)$ difference independent? More precisely, do $\zeta\left(s+s_{0}\right), \zeta\left(s+s_{1}\right), \ldots, \zeta\left(s+s_{m}\right)$ satisfy any algebraic equation or not? Here $m$ is a nonnegative integer, $s_{i}, i=0,1, \ldots, m$, are distinct complex numbers. The special case that $s_{0}, s_{1}, \ldots, s_{m}$ are real numbers was studied by Ostrowski [13]. He proved that $\zeta\left(s+s_{0}\right), \zeta\left(s+s_{1}\right), \ldots, \zeta\left(s+s_{m}\right)$ do not satisfy any algebraic equation with rational coefficients. For "small" $s_{i}$ 's, Voronin [17] proved the following.

Theorem A. Let $m$ be a nonnegative integer, and $s_{i} \in \mathbb{C}, i=0,1, \ldots, m$, $s_{i} \neq s_{j}, 0 \leq i<j \leq m$. If

$$
\left|s_{i}\right|<1 / 4, \quad i=0,1, \ldots, m,
$$

and

$$
f\left(\zeta\left(s+s_{0}\right), \zeta\left(s+s_{1}\right), \ldots, \zeta\left(s+s_{m}\right)\right)=0
$$

identically in $s \in \mathbb{C}$, where $f\left(z_{0}, z_{1}, \ldots, z_{m}\right)$ is a continuous function, then $f$ is identically zero.

Theorem A is not always true for "large" $s_{i}$ 's, since the vectors $(\zeta(s)$, $\left.\zeta\left(s+s_{0}\right)\right)$ are not dense in $\mathbb{C}^{2}$ for any given complex $s_{0}$ with $\Re s_{0}>1$, as follows from elementary properties of the zeta and gamma functions, where $\Re s_{0}$ means the real part of $s_{0}$.

In order to state our main result, we recall the standard notations of Nevanlinna theory. Let $\phi$ be a meromorphic function on the complex plane. The Nevanlinna characteristic $T(r, \phi)$ of $\phi$ for $r \geq 0$ is defined by

$$
T(r, \phi)=N(r, \phi)+m(r, \phi),
$$

where

$$
N(r, \phi)=\int_{0}^{r} \frac{n(t, \phi)-n(0, \phi)}{t} d t+n(0, \phi) \log r
$$

is the pole counting function, $n(r, \phi)$ is the number of poles (counting multiplicities) of $\phi$ in the disc $\{z:|z| \leq r\}$, and the proximity function $m(r, \phi)$ is given by

$$
m(r, \phi)=\frac{1}{2 \pi} \int_{0}^{2 \pi} \log ^{+}\left|\phi\left(r e^{\mathrm{i} \theta}\right)\right| d \theta
$$

where $\log ^{+} x=\max \{0, \log x\}$. We have

THEOREM 1. The Riemann zeta function $\zeta(s)$ does not satisfy any algebraic difference equation whose coefficients are meromorphic functions $\phi$ 
with Nevanlinna characteristic satisfying $T(r, \phi)=o(r)$ as $r \rightarrow \infty$. That is, if

$$
f\left(s, \zeta\left(s+s_{0}\right), \zeta\left(s+s_{1}\right), \ldots, \zeta\left(s+s_{m}\right)\right)=0
$$

for all $s \in \mathbb{C}$, where $m$ is a nonnegative integer, $s_{i}, i=0,1, \ldots, m$, are distinct complex numbers, and $f$ is a polynomial in $\zeta\left(s+s_{0}\right), \zeta\left(s+s_{1}\right)$, $\ldots, \zeta\left(s+s_{m}\right)$ whose coefficients are meromorphic functions $\phi(s)$ with Nevanlinna characteristic satisfying $T(r, \phi)=o(r)$ as $r \rightarrow \infty$, then $f$ is identically zero.

It is well known that a meromorphic function $\phi$ is rational if and only if $T(r, \phi)=O(\log r)$ as $r \rightarrow \infty$. Theorem 1 implies that $\zeta(s)$ does not satisfy any algebraic difference equation whose coefficients are rational functions.

We recall that the Nevanlinna order of a meromorphic function $\phi$ is defined by

$$
\varrho(\phi)=\limsup _{r \rightarrow \infty} \frac{\log T(r, \phi)}{\log r} .
$$

Theorem 1 implies

COROLlary 1. The Riemann zeta function $\zeta(s)$ does not satisfy any algebraic difference equation whose coefficients are meromorphic functions $\phi$ with Nevanlinna order $\varrho(\phi)<1$.

It is easily seen that the Nevanlinna order of $\zeta(s)$ is $\varrho(\zeta(s))=1$. By a similar calculation, we have

$$
\varrho\left(\frac{\zeta(s+1)}{\zeta(s)}\right)=1 .
$$

It follows that Corollary 1 is best possible in the sense that the condition $\varrho(\phi)<1$ cannot be relaxed to $\varrho(\phi) \leq 1$.

2. Proof of the constant coefficients case of Theorem 1 . In this section, we prove the constant coefficients case of Theorem 1.

THEOREM 2. The Riemann zeta function $\zeta(s)$ does not satisfy any algebraic difference equation with constant coefficients. That is, if

$$
f\left(\zeta\left(s+s_{0}\right), \zeta\left(s+s_{1}\right), \ldots, \zeta\left(s+s_{m}\right)\right)=0
$$

for all $s \in \mathbb{C}$, where $m$ is a nonnegative integer, $s_{i} \in \mathbb{C}, i=0,1, \ldots, m$, are distinct complex numbers, $f$ is a polynomial in $\zeta\left(s+s_{0}\right), \zeta\left(s+s_{1}\right), \ldots$, $\zeta\left(s+s_{m}\right)$ whose coefficients are complex constants, then $f$ is identically zero.

To prove Theorem 2, we need the following lemma.

Lemma 1. Let $b_{i}, s_{i} \in \mathbb{C}, i=0,1, \ldots, m, s_{i} \neq s_{j}, 0 \leq i<j \leq m$. Suppose that there exists an integer $p_{0}>0$ such that for all prime numbers $p>p_{0}$, 


$$
\sum_{i=0}^{m} b_{i} p^{-s_{i}}=0
$$

Then

$$
b_{i}=0, \quad i=0,1, \ldots, m .
$$

Proof. We construct an entire function of exponential type

$$
g(z)=\sum_{i=0}^{m} b_{i} e^{-s_{i} z}
$$

It is not difficult to see that $g(z)$ cannot have more than $c r$ zeros in a disc of radius $r$, where $c$ is a constant, unless it vanishes identically. It follows from the hypothesis $(2.2)$ that $g(z)$ vanishes at $z=\log p$ for all primes $p>p_{0}$. We deduce from the prime number theorem that the number of zeros of $g(z)$ in a disc of radius $r$ is at least

$$
e^{r} / r+o\left(e^{r} / r\right) \geq c r
$$

as $r \rightarrow \infty$. Therefore $g(z)$ is identically zero and then (2.3) follows.

Proof of Theorem 2. Assume that $\zeta(s)$ satisfies

$$
\sum_{i=1}^{N} P_{i} \zeta\left(s+s_{0}\right)^{k_{0}(i)} \zeta\left(s+s_{1}\right)^{k_{1}(i)} \cdots \zeta\left(s+s_{m}\right)^{k_{m}(i)}=0,
$$

where $P_{i}(i=1, \ldots, N)$ are complex constants, not all zero, and $K(i)=$ $\left(k_{0}(i), k_{1}(i), \ldots, k_{m}(i)\right), i=1, \ldots, N$, are multi-indices with all indices being nonnegative integers, such that

$$
K(i) \neq K(j), \quad 1 \leq i<j \leq N .
$$

Here $K(i)=K(j)$ means $k_{l}(i)=k_{l}(j)$ for all $0 \leq l \leq m$. Let

$$
\sum_{n=1}^{\infty} \frac{A_{i}(n)}{n^{s}}:=\zeta\left(s+s_{0}\right)^{k_{0}(i)} \zeta\left(s+s_{1}\right)^{k_{1}(i)} \cdots \zeta\left(s+s_{m}\right)^{k_{m}(i)},
$$

for $i=1, \ldots, N$, where the Dirichlet series is convergent in the region

$$
1-\min \left\{\Re s_{0}, \Re s_{1}, \ldots, \Re s_{m}\right\}<\Re s<\infty .
$$

Then by the uniqueness theorem for Dirichlet series, we have

$$
\sum_{i=1}^{N} P_{i} A_{i}(n)=0, \quad n=1,2, \ldots
$$

If there exists an $i^{\prime}, 1 \leq i^{\prime} \leq N$, such that $K\left(i^{\prime}\right)=(0,0, \ldots, 0)$, then there exists an $i \neq i^{\prime}$ such that $P_{i} \neq 0$. (Otherwise $P_{i^{\prime}} \neq 0$ and $P_{i^{\prime}} A_{i^{\prime}}(1)=0$; but $A_{i^{\prime}}(1)=1$, so we have a contradiction.) Since

$$
A_{i^{\prime}}(n)=0, \quad n=2,3, \ldots,
$$


we have

$$
\sum_{1 \leq i \leq N, i \neq i^{\prime}} P_{i} A_{i}(n)=0, \quad n=2,3, \ldots
$$

with $P_{i}, 1 \leq i \leq N, i \neq i^{\prime}$, not all zero.

So, we may assume without loss of generality that

$$
K(i) \neq(0,0, \ldots, 0), \quad 1 \leq i \leq N
$$

and

$$
\sum_{i=1}^{N} P_{i} A_{i}(n)=0, \quad n=2,3, \ldots
$$

with $P_{i}, i=1, \ldots, N$, not all zero.

Let $L$ be an arbitrary positive integer, and $p_{1}, \ldots, p_{L}$ be distinct prime numbers. By $(2.7)$, for $i=1, \ldots, N$ we have

$$
A_{i}\left(p_{1} \cdots p_{L}\right)=\prod_{1 \leq l \leq L}\left(k_{0}(i) p_{l}^{-s_{0}}+k_{1}(i) p_{l}^{-s_{1}}+\cdots+k_{m}(i) p_{l}^{-s_{m}}\right) .
$$

It follows from (2.8) and (2.13) that

$$
\sum_{i=1}^{N} P_{i} \prod_{1 \leq l \leq L}\left(k_{0}(i) p_{l}^{-s_{0}}+k_{1}(i) p_{l}^{-s_{1}}+\cdots+k_{m}(i) p_{l}^{-s_{m}}\right)=0 .
$$

Let $p_{1}, \ldots, p_{L-1}$ be fixed, and let $p_{L}$ vary over

$$
\left\{p \mid p \text { is a prime number, } p>\max _{1 \leq l \leq L-1} p_{l}\right\} \text {. }
$$

We then have, for all prime numbers $p>\max _{1 \leq l \leq L-1} p_{l}$,

$$
\begin{aligned}
\sum_{q_{1}=0}^{m}\left[\sum _ { i = 1 } ^ { N } P _ { i } \prod _ { 1 \leq l \leq L - 1 } \left(k_{0}(i) p_{l}^{-s_{0}}\right.\right. & +k_{1}(i) p_{l}^{-s_{1}} \\
& \left.\left.+\cdots+k_{m}(i) p_{l}^{-s_{m}}\right) k_{q_{1}}(i)\right] p^{-s_{q_{1}}}=0 .
\end{aligned}
$$

Thus by Lemma 1 , for $q_{1}=0,1, \ldots, m$,

$$
\sum_{i=1}^{N} P_{i} \prod_{1 \leq l \leq L-1}\left(k_{0}(i) p_{l}^{-s_{0}}+k_{1}(i) p_{l}^{-s_{1}}+\cdots+k_{m}(i) p_{l}^{-s_{m}}\right) k_{q_{1}}(i)=0 .
$$

Let $p_{1}, \ldots, p_{L-2}$ be fixed, and let $p_{L-1}$ vary over

$$
\left\{p \mid p \text { is a prime number, } p>\max _{1 \leq l \leq L-2} p_{l}\right\} \text {. }
$$

We then have, for $q_{1}=0,1, \ldots, m$ and all prime numbers $p>\max _{1 \leq l \leq L-2} p_{l}$,

$$
\begin{aligned}
\sum_{q_{2}=0}^{m}\left[\sum _ { i = 1 } ^ { N } P _ { i } \prod _ { 1 \leq l \leq L - 2 } \left(k_{0}(i) p_{l}^{-s_{0}}+k_{1}(i) p_{l}^{-s_{1}}\right.\right. \\
\left.\left.+\cdots+k_{m}(i) p_{l}^{-s_{m}}\right) k_{q_{1}}(i) k_{q_{2}}(i)\right] p^{-s_{q_{2}}}=0
\end{aligned}
$$


Thus by Lemma 1 again, for $q_{1}, q_{2}=0,1, \ldots, m$,

$$
\begin{aligned}
\sum_{i=1}^{N} P_{i} \prod_{1 \leq l \leq L-2}\left(k_{0}(i) p_{l}^{-s_{0}}+k_{1}(i) p_{l}^{-s_{1}}\right. & \\
& \left.+\cdots+k_{m}(i) p_{l}^{-s_{m}}\right) k_{q_{1}}(i) k_{q_{2}}(i)=0 .
\end{aligned}
$$

Continuing this procedure, we finally have, for $L=1,2, \ldots$,

$$
\sum_{i=1}^{N} P_{i} k_{q_{1}}(i) \cdots k_{q_{L}}(i)=0, \quad q_{1}, \ldots, q_{L}=0,1, \ldots, m .
$$

Then

$$
\begin{aligned}
\sum_{i=1}^{N} P_{i}\left(k_{0}(i)+k_{1}(i)\right. & \left.x+\cdots+k_{m}(i) x^{m}\right)^{L} \\
= & \sum_{i=1}^{N} P_{i} \sum_{0 \leq q_{1}, \ldots, q_{L} \leq m} k_{q_{1}}(i) \cdots k_{q_{L}}(i) x^{q_{1}+\cdots+q_{L}} \\
& =\sum_{0 \leq q_{1}, \ldots, q_{L} \leq m} x^{q_{1}+\cdots+q_{L}} \sum_{i=1}^{N} P_{i} k_{q_{1}}(i) \cdots k_{q_{L}}(i) \\
& =0, \quad \forall x \in \mathbb{R}, L=1,2, \ldots
\end{aligned}
$$

Define

$$
\begin{aligned}
& H_{i}(x)=k_{0}(i)+k_{1}(i) x+\cdots+k_{m}(i) x^{m}, \quad i=1, \ldots, N, \\
& H_{0}(x)=0 .
\end{aligned}
$$

Noticing that $P_{i}, i=1, \ldots, N$, are not all zero, by a well known theorem on determinants due to Vandermonde, for every $x \in \mathbb{R}$ there exist $0 \leq i_{1}(x)<$ $i_{2}(x) \leq N$ such that

$$
H_{i_{1}(x)}(x)=H_{i_{2}(x)}(x)
$$

Let

$$
E_{i j}=\left\{x \mid H_{i}(x)=H_{j}(x)\right\}, \quad 0 \leq i<j \leq N .
$$

Then

$$
\bigcup_{0 \leq i<j \leq N} E_{i j}=\mathbb{R}
$$

Hence there must exist $0 \leq \widehat{i}<\widehat{j} \leq N$ such that $E_{\widehat{i} \widehat{j}}$ is infinite. That is, the polynomial

$$
H_{\widehat{i}}(x)-H_{\widehat{j}}(x)
$$

has infinitely many zeros, so it is the zero polynomial, which contradicts (2.6) and (2.11). The proof is complete. 
3. Proof of Theorem 1. We need the following well known lemma of Cartan [2] (see also [8]).

Lemma 2. Let $z_{1}, \ldots, z_{p}$ be any finite collection of complex numbers, and let $B>0$ be any given positive number. Then there exists a finite collection of closed discs $D_{1}, \ldots, D_{q}$ with respective radii $r_{1}, \ldots, r_{q}$ that satisfy

$$
r_{1}+\cdots+r_{q}=2 B
$$

such that if $z \notin D_{j}$ for $j=1, \ldots, q$, then there is a permutation of the points $z_{1}, \ldots, z_{p}$, say $\widehat{z}_{1}, \ldots, \widehat{z}_{p}$, that satisfies

$$
\left|z-\widehat{z}_{\mu}\right|>B \mu / p, \quad \mu=1, \ldots, p,
$$

where the permutation may depend on $z$.

The following two lemmas may be of independent interest.

Lemma 3. Let $\phi$ be a meromorphic function, and let $\alpha>1, \varepsilon>0$ be given real constants. Then there exists a set $E \subset(1, \infty)$ that has upper logarithmic density

$$
\delta(E):=\limsup _{x \rightarrow \infty} \frac{\int_{E \cap(1, x]}(1 / t) d t}{\log x}<\varepsilon
$$

and a constant $A>0$ such that for all $z$ satisfying $|z|=r \notin[0,1] \cup E$, we have

$$
|\phi(z)| \leq e^{A T(\alpha r, \phi)} .
$$

REMARK. Although an upper bound similar to (3.4) for the meromorphic function $\phi(z)$ outside an exceptional set should have been recorded in the literature, the authors have not been able to find such a reference.

Proof. Assume $\phi$ is not identically zero. Let $\left(a_{\nu}\right)_{\nu \in \mathbb{N}}$, resp. $\left(b_{\mu}\right)_{\mu \in \mathbb{N}}$, denote the sequence of all zeros, resp. all poles, of $\phi$, with due account of multiplicity. By the Poisson-Jensen formula, for $|z|=r<R$ we have

$$
\begin{aligned}
\log |\phi(z)|= & \frac{1}{2 \pi} \int_{0}^{2 \pi} \log \left|\phi\left(R e^{\mathrm{i} \theta}\right)\right| \Re\left(\frac{R e^{\mathrm{i} \theta}+z}{R e^{\mathrm{i} \theta}-z}\right) d \theta \\
& -\sum_{\left|a_{\nu}\right|<R} \log \left|\frac{R^{2}-\bar{a}_{\nu} z}{R\left(z-a_{\nu}\right)}\right|+\sum_{\left|b_{\mu}\right|<R} \log \left|\frac{R^{2}-\bar{b}_{\mu} z}{R\left(z-b_{\mu}\right)}\right| \\
\leq & \frac{R+r}{R-r} \cdot \frac{1}{2 \pi} \int_{0}^{2 \pi}|\log | \phi\left(R e^{\mathrm{i} \theta}\right)|| d \theta+\sum_{\left|b_{\mu}\right|<R} \log \left|\frac{R^{2}-\bar{b}_{\mu} z}{R\left(z-b_{\mu}\right)}\right| \\
\leq & \frac{R+r}{R-r}(m(R, \phi)+m(R, 1 / \phi))+\sum_{\left|b_{\mu}\right|<R} \log \frac{R+r}{\left|z-b_{\mu}\right|},
\end{aligned}
$$


where we have used the estimate (see [4])

$$
\left|\frac{R^{2}-\bar{a} z}{R(z-a)}\right|>1 \quad \text { for }|z|<R,|a|<R .
$$

Letting $R=\alpha^{1 / 3} r, \alpha>1$, we get

$$
\begin{aligned}
\log |\phi(z)| & \leq A_{1} T\left(\alpha^{1 / 3} r, \phi\right)+\sum_{\left|b_{\mu}\right|<\alpha^{1 / 3} r} \log \frac{\left(\alpha^{1 / 3}+1\right) r}{\left|z-b_{\mu}\right|} \\
& \leq A_{1} T(\alpha r, \phi)+\sum_{\left|b_{\mu}\right|<\alpha^{1 / 3} r} \log \frac{\left(\alpha^{1 / 3}+1\right) r}{\left|z-b_{\mu}\right|}
\end{aligned}
$$

where $A_{1}>0$ is a constant.

Now we estimate the last sum. We suppose that $h$ is a fixed nonnegative integer, and that $z$ is confined to the annulus

$$
\alpha^{h / 3} \leq|z|=r \leq \alpha^{(h+1) / 3} \text {. }
$$

Setting $p=n\left(\alpha^{(h+2) / 3}, \phi\right), B=\varepsilon \alpha^{h / 3}$, and applying Lemma 2 to the points $b_{1}, \ldots, b_{p}$, we find that there exists a finite collection of closed discs $D_{1}, \ldots, D_{q}$, whose radii add up to $2 B$, such that if $z \notin D_{j}$ for $j=1, \ldots, q$, then there is a permutation of the points $b_{1}, \ldots, b_{p}$, say $\widehat{b}_{1}, \ldots, \widehat{b}_{p}$, such that

$$
\left|z-\widehat{b}_{\mu}\right|>B \mu / p, \quad \mu=1, \ldots, p .
$$

Notice that here $q$ and $D_{1}, \ldots, D_{q}$ depend on $p$ and so on $h$. Hence if $z \notin D_{j}$ for $j=1, \ldots, q$, we deduce from (3.8) and (3.9) that

$$
\begin{array}{r}
\sum_{\left|b_{\mu}\right|<\alpha^{1 / 3} r} \log \frac{\left(\alpha^{1 / 3}+1\right) r}{\left|z-b_{\mu}\right|} \leq \sum_{\mu=1}^{p} \log \frac{\left(\alpha^{1 / 3}+1\right) r}{\left|z-b_{\mu}\right|}=\sum_{\mu=1}^{p} \log \frac{\left(\alpha^{1 / 3}+1\right) r}{\left|z-\widehat{b}_{\mu}\right|} \\
\leq \sum_{\mu=1}^{p} \log \frac{\left(\alpha^{1 / 3}+1\right) r p}{B \mu} \leq \sum_{\mu=1}^{p} \log \frac{\alpha^{1 / 3}\left(\alpha^{1 / 3}+1\right) p}{\varepsilon \mu}
\end{array}
$$

Setting $A_{2}=\alpha^{1 / 3}\left(\alpha^{1 / 3}+1\right) / \varepsilon$ for simplicity, by Stirling's formula and (3.8) we have

$$
\begin{aligned}
\sum_{\left|b_{\mu}\right|<\alpha^{1 / 3} r} \log \frac{\left(\alpha^{1 / 3}+1\right) r}{\left|z-b_{\mu}\right|} & \leq \sum_{\mu=1}^{p} \log \frac{A_{2} p}{\mu}=\log \frac{\left(A_{2} p\right)^{p}}{\Gamma(p+1)} \\
& \leq \log \frac{\left(A_{2} p\right)^{p}}{A_{3} p^{1 / 2}(p / e)^{p}}=\log \frac{\left(A_{2} e\right)^{p}}{A_{3} p^{1 / 2}} \\
& \leq \log A_{4}^{p}=p \log A_{4}=n\left(\alpha^{(h+2) / 3}, \phi\right) \log A_{4} \\
& \leq A_{5} n\left(\alpha^{2 / 3} r, \phi\right),
\end{aligned}
$$

where $A_{3}>0, A_{4}>1, A_{5}>0$ are constants independent of $r$. For $\alpha^{2 / 3} r>1$, 
we have

$$
\begin{aligned}
N(\alpha r, \phi) & \geq \int_{\alpha^{2 / 3} r}^{\alpha r} \frac{n(t, \phi)-n(0, \phi)}{t} d t+n(0, \phi) \log (\alpha r) \\
& \geq n\left(\alpha^{2 / 3} r, \phi\right) \int_{\alpha^{2 / 3} r}^{\alpha r} \frac{d t}{t}-n(0, \phi) \int_{\alpha^{2 / 3} r}^{\alpha r} \frac{d t}{t}+n(0, \phi) \log (\alpha r) \\
& \geq n\left(\alpha^{2 / 3} r, \phi\right) \frac{\alpha r-\alpha^{2 / 3} r}{\alpha r}=\left(1-\alpha^{-1 / 3}\right) n\left(\alpha^{2 / 3} r, \phi\right),
\end{aligned}
$$

so that

$$
n\left(\alpha^{2 / 3} r, \phi\right) \leq \frac{1}{1-\alpha^{-1 / 3}} N(\alpha r, \phi) .
$$

Now (3.11) and (3.13) yield

$$
\sum_{\left|b_{\mu}\right|<\alpha^{1 / 3} r} \log \frac{\left(\alpha^{1 / 3}+1\right) r}{\left|z-b_{\mu}\right|} \leq A_{6} N(\alpha r, \phi) \leq A_{6} T(\alpha r, \phi)
$$

with a constant $A_{6}>0$.

For each $h$, we define (recall that $q$ and $D_{1}, \ldots, D_{q}$ depend on $h$ )

$$
Y_{h}=\left\{r \mid \text { there exists } z \in \bigcup_{j=1}^{q} D_{j} \text { such that }|z|=r\right\} \text {, }
$$

Then

$$
\int_{E_{h}} 1 d x \leq \int_{Y_{h}} 1 d x \leq 4 B=4 \varepsilon \alpha^{h / 3}
$$

Set

$$
E=\bigcup_{h=0}^{\infty} E_{h} \cap(1, \infty)
$$

Then by (3.7) and (3.14), for all $z$ satisfying $|z|=r \notin[0,1] \cup E$ we have

$$
|\phi(z)| \leq e^{A T(\alpha r, \phi)}
$$

with $A=A_{1}+A_{6}$.

For any $x>1$, there exists a nonnegative integer $h$ such that

$$
\alpha^{h / 3}<x \leq \alpha^{(h+1) / 3} .
$$

It follows from (3.20) and (3.17) that

$$
\begin{aligned}
\int_{E \cap(1, x]} \frac{1}{t} d t & \leq \int_{E \cap\left(1, \alpha^{(h+1) / 3}\right]} \frac{1}{t} d t=\sum_{j=0}^{h} \int_{E_{j}} \frac{1}{t} d t \leq \sum_{j=0}^{h} \frac{1}{\alpha^{j / 3}} 4 \varepsilon \alpha^{j / 3} \\
& =4 \varepsilon(h+1) \leq 12 \varepsilon \frac{\log x}{\log \alpha}+4 \varepsilon .
\end{aligned}
$$


Therefore

$$
\delta(E)=\limsup _{x \rightarrow \infty} \frac{\int_{E \cap(1, x]}(1 / t) d t}{\log x}<\frac{12 \varepsilon}{\log \alpha} .
$$

Since $\varepsilon$ is arbitrarily small, the proof is complete.

Lemma 4. Let $N$ be a positive integer. Suppose that the Dirichlet series

$$
F_{i}(s)=\sum_{n=1}^{\infty} \frac{a_{i}(n)}{n^{s}}, \quad i=1, \ldots, N
$$

are convergent in the region $\sigma_{0}<\sigma<\infty$, and for each $i=1, \ldots, N, \phi_{i}(s)$ is meromorphic function in the complex plane with Nevanlinna characteristic satisfying

$$
T\left(r, \phi_{i}\right)=o(r) \quad \text { as } r \rightarrow \infty .
$$

Suppose that

$$
\sum_{i=1}^{N} \phi_{i}(s) F_{i}(s)=0
$$

identically in $\sigma_{0}<\sigma<\infty$. Then for each positive integer $n$,

$$
\sum_{i=1}^{N} a_{i}(n) \phi_{i}(s)=0
$$

identically in the complex plane.

Proof. We argue by contradiction. Let $n_{0}$ be the minimal index for which

$$
\sum_{i=1}^{N} a_{i}\left(n_{0}\right) \phi_{i}(s)
$$

is not identically zero in the complex plane. Then by (3.23) and (3.25),

$$
\left[\sum_{i=1}^{N} a_{i}\left(n_{0}\right) \phi_{i}(s)\right] \frac{1}{n_{0}^{s}}+\sum_{i=1}^{N} \phi_{i}(s)\left[\sum_{n=n_{0}+1}^{\infty} \frac{a_{i}(n)}{n^{s}}\right]=0
$$

identically in $\sigma_{0}<\sigma<\infty$. Define

$$
\psi_{i}(s)=\frac{\phi_{i}(s)}{\sum_{i=1}^{N} a_{i}\left(n_{0}\right) \phi_{i}(s)}, \quad i=1, \ldots, N .
$$

Then

$$
-1=\sum_{i=1}^{N} \psi_{i}(s) n_{0}^{s}\left[\sum_{n=n_{0}+1}^{\infty} \frac{a_{i}(n)}{n^{s}}\right]
$$


identically in $\sigma_{0}<\sigma<\infty$. By using the well known properties of the Nevanlinna characteristic, we infer from (3.24) and (3.29) that

$$
T\left(r, \psi_{i}\right)=o(r) \quad \text { as } r \rightarrow \infty, \quad i=1, \ldots, N .
$$

Then by using Lemma 3 with $\varepsilon$ small enough, we deduce that there exists a real sequence $\max \left\{0, \sigma_{0}\right\}<\sigma_{k} \rightarrow \infty$ such that for each $\epsilon>0$, there exists $k_{\epsilon}>0$ such that for $k>k_{\epsilon}$,

$$
\left|\psi_{i}\left(\sigma_{k}\right)\right| \leq e^{\epsilon \sigma_{k}}, \quad i=1, \ldots, N .
$$

By the general theory of Dirichlet series, there exists $M>0$ independent of $\sigma_{k}$ such that

$$
\left|\sum_{n=n_{0}+1}^{\infty} \frac{a_{i}(n)}{n^{\sigma_{k}}}\right| \leq M\left(n_{0}+1\right)^{-\sigma_{k}}, \quad k>k_{\epsilon}, i=1, \ldots, N .
$$

By (3.30), (3.32) and (3.33), for $k>k_{\epsilon}$ we have

$$
\begin{aligned}
1=\left|\sum_{i=1}^{N} \psi_{i}\left(\sigma_{k}\right) n_{0}^{\sigma_{k}}\left[\sum_{n=n_{0}+1}^{\infty} \frac{a_{i}(n)}{n^{\sigma_{k}}}\right]\right| & \leq N M e^{\epsilon \sigma_{k}}\left(\frac{n_{0}}{n_{0}+1}\right)^{\sigma_{k}} \\
& =N M e^{\left(\epsilon-\log \frac{n_{0}+1}{n_{0}}\right) \sigma_{k}} .
\end{aligned}
$$

Now we may choose $\epsilon$ such that $0<\epsilon<\log \frac{n_{0}+1}{n_{0}}$; then the right hand side of (3.34) tends to zero as $k \rightarrow \infty$ and we have a contradiction.

Proof of Theorem 1. Assume that $\zeta(s)$ satisfies the difference equation

$$
\sum_{i=1}^{N} \phi_{i}(s) \zeta\left(s+s_{0}\right)^{k_{0}(i)} \zeta\left(s+s_{1}\right)^{k_{1}(i)} \cdots \zeta\left(s+s_{m}\right)^{k_{m}(i)}=0,
$$

where $\phi_{i}(s), i=1, \ldots, N$, are meromorphic functions not all identically zero in the complex plane, with Nevanlinna characteristics satisfying

$$
T\left(r, \phi_{i}\right)=o(r) \quad \text { as } r \rightarrow \infty,
$$

and $K(i)=\left(k_{0}(i), k_{1}(i), \ldots, k_{m}(i)\right), i=1, \ldots, N$, are multi-indices with every index being a nonnegative integer such that

$$
K(i) \neq K(j), \quad 1 \leq i<j \leq N .
$$

Let

$$
\begin{array}{r}
\sum_{n=1}^{\infty} \frac{A_{i}(n)}{n^{s}}:=\zeta\left(s+s_{0}\right)^{k_{0}(i)} \zeta\left(s+s_{1}\right)^{k_{1}(i)} \cdots \zeta\left(s+s_{m}\right)^{k_{m}(i)} \\
i=1, \ldots, N
\end{array}
$$

where the Dirichlet series is convergent in the region

$$
1-\min \left\{\Re s_{0}, \Re s_{1}, \ldots, \Re s_{m}\right\}<\Re s<\infty .
$$


By Lemma 4, for each positive integer $n$,

$$
\sum_{i=1}^{N} A_{i}(n) \phi_{i}(s)=0
$$

identically in the complex plane.

Let $S$ be a fixed point such that not all $\phi_{i}(S), i=1, \ldots, N$, are zero. Then

$$
\sum_{i=1}^{N} A_{i}(n) \phi_{i}(S)=0, \quad n=1,2, \ldots
$$

We deduce from (3.40) and (3.38) that

$$
\sum_{i=1}^{N} \phi_{i}(S) \zeta\left(s+s_{0}\right)^{k_{0}(i)} \zeta\left(s+s_{1}\right)^{k_{1}(i)} \cdots \zeta\left(s+s_{m}\right)^{k_{m}(i)}=0
$$

identically in the complex plane. That is, $\zeta(s)$ satisfies a nontrivial difference equation with constant coefficients. This contradicts Theorem 2 , and completes the proof.

Acknowledgements. The authors would like to express their sincere thanks to the referee for his valuable comments, and in particular for shortening the original proof to Lemma 1, which greatly improved the readability of the paper.

\section{References}

[1] S. B. Bank and R. P. Kaufman, An extension of Hölder's theorem concerning the Gamma function, Funkcial. Ekvac. 19 (1976), 53-63.

[2] H. Cartan, Sur les systèmes de fonctions holomorphes à variétés linéaires lacunaires et leurs applications, Ann. Sci. École Norm. Sup. (3) 45 (1928), 255-346.

[3] F. Hausdorff, Zum Hölderschen Satz über $\Gamma(z)$, Math. Ann. 94 (1925), 244-247.

[4] W. K. Hayman, Meromorphic Functions, Oxford Univ. Press, Oxford, 1964.

[5] D. Hilbert, Mathematische Probleme, in: Die Hilbertschen Probleme, Geest \& Portig, Leipzig, 1971, 23-80.

[6] O. Hölder, Über die Eigenschaft der $\Gamma$-Function, keiner algebraischen Differentialgleichung zu genügen, Math. Ann. 28 (1887), 1-13.

[7] A. A. Karatsuba and S. M. Voronin, The Riemann Zeta-Function, transl. from the Russian by N. Koblitz, de Gruyter, Berlin, 1992.

[8] B. Ja. Levin, Distribution of Zeros of Entire Functions, rev. ed., transl. from the Russian by R. P. Boas et al., Amer. Math. Soc., Providence, 1980.

[9] E. Moore, Concerning transcendentally transcendental functions, Math. Ann. 48 (1897), 49-74.

[10] D. D. Mordykhai-Boltovskoi, On hypertranscendence of the function $\xi(x, s)$, Izv. Politekh. Inst. Varsh. 2 (1914), 1-16. 
[11] A. Ostrowski, Neuer Beweis des Hölderschen Satzes, daß die $\Gamma$-Function keiner algebraischen Differentialgleichung genügt, Math. Ann. 79 (1919), 286-288.

[12] -, Zum Hölderschen Satz über $\Gamma(x)$, ibid. 94 (1925), 248-251.

[13] - Über Dirichletsche Reihen und algebraische Differentialgleichungen, Math. Z. 8 (1920), 241-298.

[14] A. G. Postnikov, On the differential independence of Dirichlet series, Dokl. Akad. Nauk SSSR 66 (1949), 561-564 (in Russian).

[15] - Generalization of one of the Hilbert problems, ibid. 107 (1956), 512-515 (in Russian).

[16] E. C. Titchmarsh, The Theory of the Riemann Zeta Function, 2nd ed., rev. by D. R. Heath-Brown, Clarendon Press, Oxford, 1986.

[17] S. M. Voronin, The distribution of the nonzero values of the Riemann zeta-function, Trudy Mat. Inst. Steklov. 128 (1972), 131-150, 260 (in Russian).

Department of Mathematics

Hong Kong University

of Science and Technology

Clear Water Bay, Kowloon

Hong Kong, P.R. China

E-mail: machiang@ust.hk
Academy of Mathematics and Systems Science Chinese Academy of Sciences Beijing, 100080, P.R. China E-mail: fsj@amss.ac.cn

Received on 5.7.2005

and in revised form on 20.6.2006 